\title{
二维准晶结构排列树枝状单元左手材料的 电磁响应行为
}

\author{
周 欣 赵晓鹏* \\ (西北工业大学电流变技术研究所, 西安 710072.*联系人, E-mail: xpzhao@nwpu.edu.cn)
}

\begin{abstract}
摘要 提出了一种以二维准周期晶格分布树枝结构负磁导率材料模型, 研究了电磁波以不同角度入射 于周期和准晶分布的树枝状结构负磁导率材料及其与金属丝阵列组合的左手材料的微波透射及反射行 为. 结果表明, 以准晶分布的负磁导率材料以及左手材料的谐振峰随微波入射角度变化的影响较小, 并 且准晶分布树枝单元间耦合模式的改变, 能增强或减弱整体的谐振特性, 为实现各向同性左手材料提 供了新的途径.
\end{abstract}

\section{关键词 左手材料 树枝结构单元 准晶}

最近，左手材料(left-handed materials)以其特异 的物理性能和广阔的应用前景吸引了许多研究. 左 手材料是由Veselago ${ }^{[1]}$ 首先从理论上提出的一种同时 具有负介电常数和负磁导率的材料, 电磁波在其中 传播时, $E, H$ 和 $k 3$ 个矢量服从左手法则, 然而自然界 中并没有发现这种材料. Pendry等人 ${ }^{[2,3]}$ 提出周期排 列的金属丝以及开口谐振环可以分别实现负 $\varepsilon$ 和负 $\mu$ 的理论模型, Shelby等人 ${ }^{[4]}$ 通过人工制备并复合这两 种结构首次得到了微波段具有反常电磁特性的材料. 目前, 左手材料的研究已经从最初的微波频段拓展 到可见光波段, 其特殊的物理行为极大促进了完美

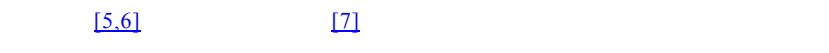
研究. 谐振单元作为左手材料微观结构的本质是重 新设计材料, 我们曾提出一种开口六边形环结构单 元 ${ }^{[8,9]}$, 并且研究了缺陷效应对负磁导率和左手行为的 影响 ${ }^{[10 \sim 12]}$. 为了实现红外或可见光波段左手性能, 研 究者提出许多简化谐振模式，如“网格”模型 [13]、“杆 对”模型 [14]等. 通过化学制备获得左手材料是备受关 注的问题, 我们率先提出了树枝结构模型, 并且从理 论和实验上证明了微波和红外波段树枝结构的左手 行为 $[15 \sim 77$. 然而, 目前的左手材料基本上都是各向 异性, 要实现各向同性左手材料, 仍有不少的困难 [18,19].

在晶体或是光子晶体中随准晶的旋转对称重数 的增加, 其各向异性减少已经为人们所熟知. 但是, 在左手材料中通常的开口谐振环是一种不对称结构,
因此能否表现出随旋转对称性的增加而出现各向异 性减少, 并非可以直接推出. 由于树枝结构自身拥有 高度的对称性, 本文利用树枝单元结构, 结合准晶材 料的多重旋转对称性, 提出了一种以二维准周期晶 格分布树枝结构负磁导率材料模型, 研究了多角度 入射情况下, 准晶分布的树枝单元结构负磁导率材 料及左手材料的微波透射、反射行为, 试图为实现各 向同性左手材料提供新的途径.

\section{1 样品制备与实验装置}

准晶的设计有多种理论方法, 本文利用匹配拼 砌法则(matching rule), 设计了四重准晶、八重准晶和 十二重准晶 3 种准晶分布. 实验中树枝状负磁导率样 品的制备采用标准的印刷电路板刻蚀技术, 在厚度 为 $1 \mathrm{~mm}$, 大小为 $65 \mathrm{~mm} \times 65 \mathrm{~mm}$ 的环氧玻璃纤维介 质基板 (介电常数 4.65)的单面刻蚀树枝结构单元, 树 枝的几何尺寸参考文献 [16]，由中心到外围的各级分 支长度为 $1.4,0.8$ 和 $1 \mathrm{~mm}$. 单元的排列方式分别为周 期排列(晶格常数 $l=9 \mathrm{~mm}$ ), 三角晶格排列(三角形边 长为 $9 \mathrm{~mm}$ ), 四重准晶 (由 $45^{\circ}$ 角的菱形和正方形堆砌 而成, 边长均为 $9 \mathrm{~mm}$ ), 八重准晶(由 $45^{\circ}$ 角的菱形和 正方形堆砌而成, 边长均为 $9 \mathrm{~mm}$ ), 十二重准晶(由等 边三角形和正方形堆砌而成, 边长均为 $9 \mathrm{~mm}$ ), 制备 的样品如图 1. 将样品固定于泡沫平台上(介电常数 近似为 1), 同时在实验过程中, 样品可以以基板的法 线方向为轴旋转. 利用AV3618 矢量分析仪和两个 $x$ 
波段喇叭分别作为发射和接收装置(图 2). 测试的环 境位于微波暗室中, 电磁波沿样品的平面入射, 电场 方向平行于样品平面. 测量样品的摆放为 $0^{\circ}, 30^{\circ}$ 和 $45^{\circ} 3$ 个状态, 即电磁波分别沿 $0^{\circ}, 30^{\circ}$ 和 $45^{\circ} 3$ 个角度 入射时, 样品的透射、相位及反射行为.

\section{2 实验结果与分析}

\section{1 准晶负磁导率材料的多角度入射}

作为负磁导率的谐振单元, 晶格常数的改变对 材料的微波频率以及响应强度是有影响的 [20], 虽然 在样品设计时我们将各种排布方式的样品晶格常数
均设为 $9 \mathrm{~mm}$, 但是准晶的样品实际上有一部分单元 间距不等于 $9 \mathrm{~mm}$. 因此, 我们首先测量了样品正常 摆放状态，即微波 $0^{\circ}$ 入射情况下的透射图谱. 从图 3(a)得出, 周期排列的样品在 $8.45 \mathrm{GHz}$ 处产生峰值为 $-15.07 \mathrm{~dB}$ 的谐振峰. 改变样品结构单元的排列方式 后, 由透射曲线可以看出各组准晶和三角晶格样品 仍在测试频段内发生谐振, 只是谐振峰的频率和强 度略有不同. 三角形晶格和十二重准晶样品的谐振 峰频率及强度变化较小, 频率向低频偏移在 $500 \mathrm{MHz}$ 以内, 强度均减小. 十二重准晶样品的谐振强度比周 期排列样品减小了 $3 \mathrm{~dB}$, 为 5 组样品中的最小值. 四

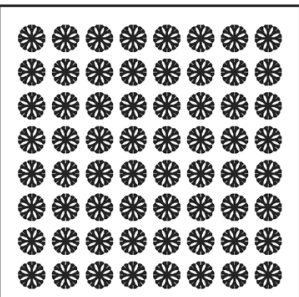

(a)

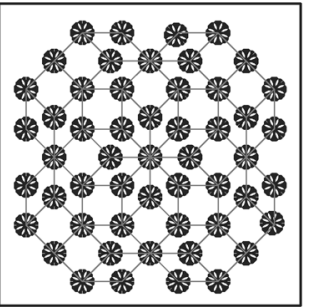

(d)

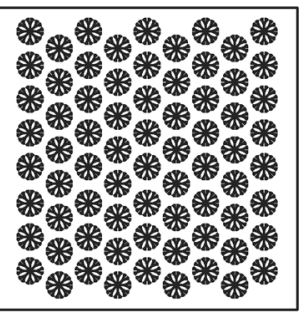

(b)

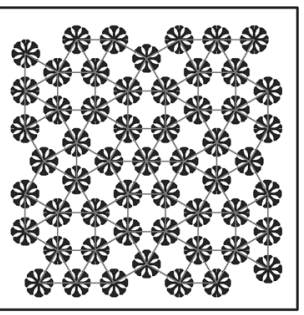

(e)

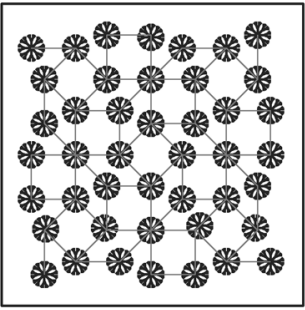

(c)

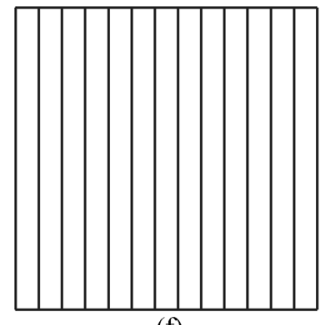

(f)

图 1 各组负磁导率样品及金属丝阵列样品

(a) 周期排列; (b) 三角晶格; (c) 四重准晶; (d) 八重准晶; (e) 十二重准晶; (f) 杆
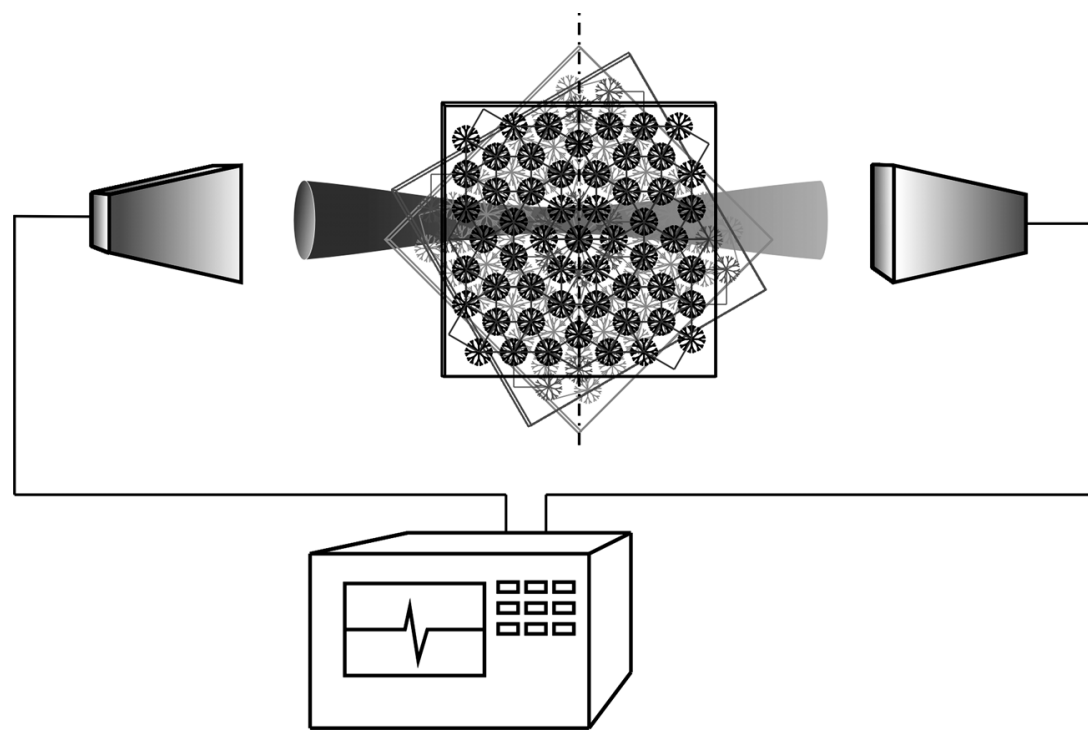

图 2 实验装置示意图 
重和八重准晶样品的谐振峰变化范围较大, 频率向 高频移动了 $1 \mathrm{GHz}$, 且谐振强度显著增强, 四重准晶 样品的强度为 $-27.89 \mathrm{~dB}$, 为 5 组样品中的最大值. 对 比相位图谱, 如图 4(a), 四重准晶样品在谐振处的相 位发生了 $166^{\circ}$ 的转变, 而周期样品仅有 $95^{\circ}$ 的转变; 同时从反射曲线图 4(b)的对比可以看出, 四重准晶样 品的反射为 $-5.4 \mathrm{~dB}$ 同样大于周期样品. 以上结果表 明, 改变结构单元的排列方式对负磁导率材料的谐 振频率有明显影响. 对于准晶排列, 由于混合了多种 单元间距, 所以单元间电磁耦合模式相比较于周期 排列有很大的不同, 可能增强或削弱其整体谐振. 接 下来, 我们对各组样品在不同角度摆放状态下分别 进行了微波透射测量. 图 3(b)为周期排布样品微波沿 $0^{\circ}, 30^{\circ}$ 和 $45^{\circ}$ 入射时的透射曲线. 可以得出, 随着微 波入射角度的增加, 材料的谐振峰频率向高频移动 约 $200 \mathrm{MHz}$, 强度减小约 $4.5 \mathrm{~dB}$. 图 3(c)为三角形晶
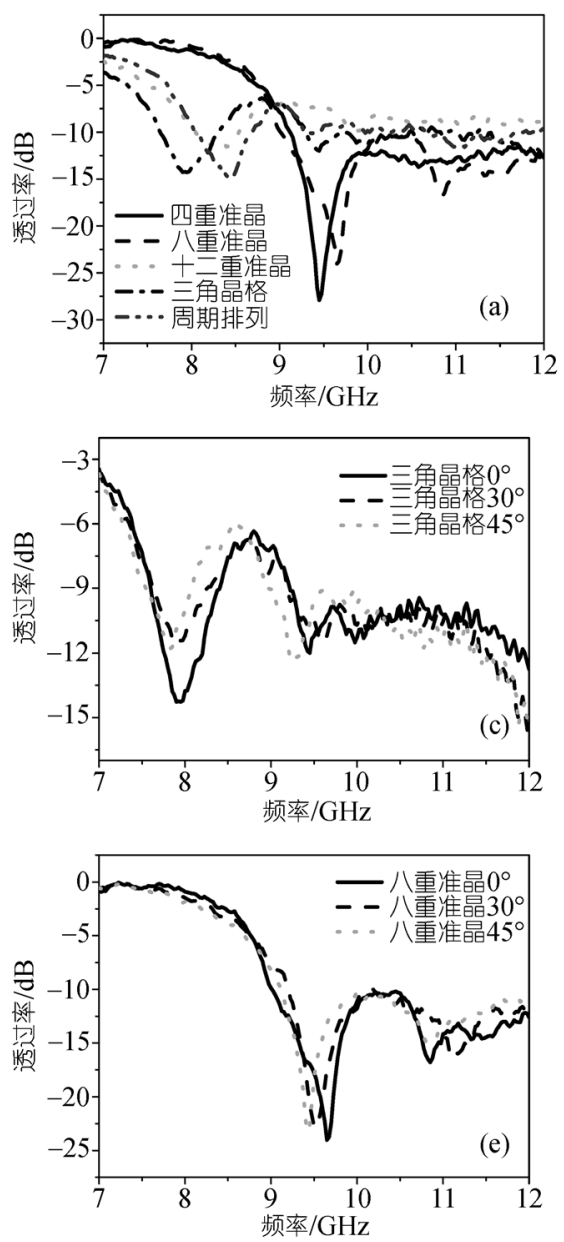

格排布样品, 随入射角度变大, 其谐振向低频移动约 $150 \mathrm{MHz}$, 强度减小约 $2.8 \mathrm{~dB}$. 图 3(d) (f)分别为四 重、八重和十二重准晶排列样品的测量结果. 可以很 明显得出, 随着单元对称重数的增加, 材料的谐振频 率和强度对微波入射角度的变化越不敏感, 如图 3(f) 的十二重准晶, 不同入射角度下其透射曲线在谐振 峰处几乎是重合的.

对于不同角度入射情况可以看作是在同一入射 角度下, 样品的晶格常数及排列方式发生变化. 如对 于周期样品，入射波矢角度变大，相当于沿波矢单元 列间距变小, $45^{\circ}$ 时类似于样品单元直角三角形晶格 排列, 因此谐振频率向高频移动; 而对于三角晶格样 品, 入射波矢角度变大, 相当于沿波矢单元列间距变 大, 在 $45^{\circ}$ 时单元排布接近周期排布, 所以频率向低 频移动. 而对于准晶材料, 由于其特殊的多重旋转对 称性, 随着对称重数增加, 微波以不同角度入射时,
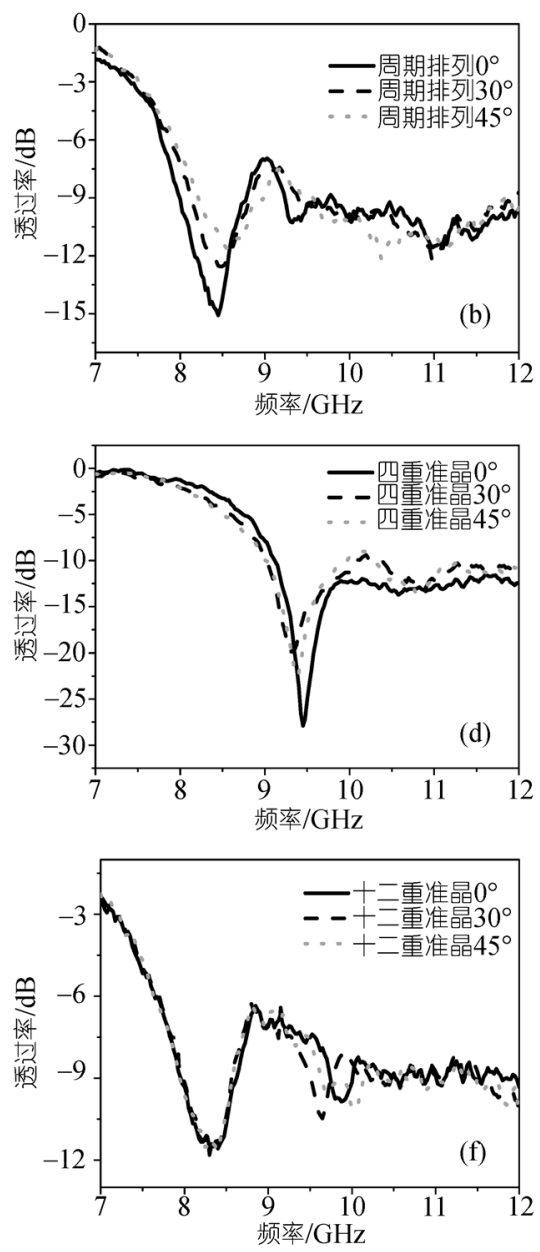

图 3 ht入射角度分别为 $0^{\circ}, 30^{\circ}$ 和 $45^{\circ}$ 时各组负磁导率材料的透射谱图 
沿波矢经过的单元晶格变化越小, 谐振峰对角度变 化越不敏感.

\section{2 准晶左手复合材料的多角度入射}

将各组负磁导率材料与周期排列的金属丝复合, 当两者的谐振区域重叠时, 就可以得到左手复合材 料. 实验中所用金属丝阵列的几何参数为长 $65 \mathrm{~mm}$,
线宽 $0.5 \mathrm{~mm}$, 间距 $5 \mathrm{~mm}$, 所用介质基板与负磁导率 材料基板相同，两基板间距 $5 \mathrm{~mm}$. 同样，我们首先测 试的是正常入射时的各组左手复合材料微波透射行 为. 如图 5(a), 在 7 10 GHz 频段内, 仅放置金属丝样 品测得的是 $-10 \mathrm{~dB}$ 左右的禁带, 即在此范围内金属 丝样品具有负的介电常数. 测试的各组复合样品
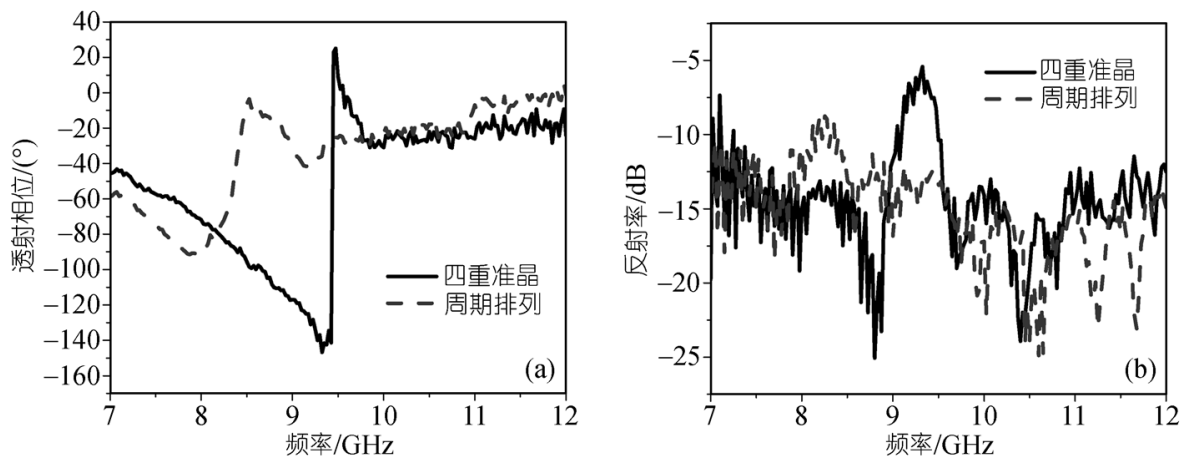

图 4 入射角度为 0 时, 周期样品及四重准晶样品的透射相位谱图(a)和反射谱图(b)
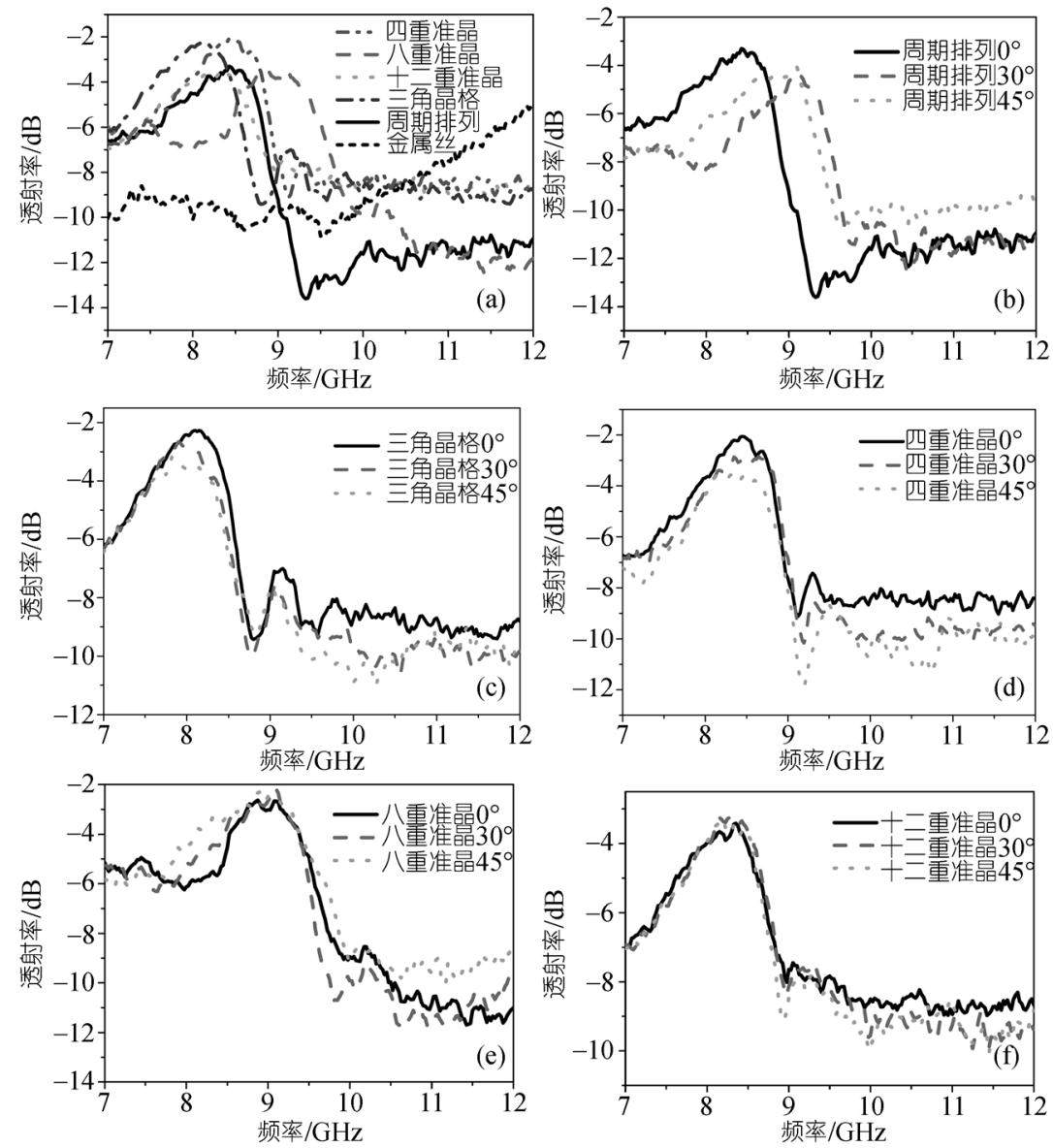

图 5 入射角度分别为 $0^{\circ}, 30^{\circ}$ 和 $45^{\circ}$ 时各组左手复合材料的透射谱图 
均在原负磁导率谐振峰附近产生左手透射峰, 其中 四重准晶的透射峰值最高, 为 $-2.07 \mathrm{~dB}$. 改变左手复 合材料的摆放角度(带有金属丝的基板角度不变, 转 动负磁导率材料的基板)，图 5(b) (f)为测试的结果. 与前文的结论类似，周期排列的左手样品的透射峰 随微波入射角度的变化影响较大; 而随着准晶样品 对称重数的增加, 其透射峰随入射角度变化的影响 逐渐减小.

\section{3 结论}

本文分别制备了周期排列、三角晶格排列以及 四重准晶、八重准晶和十二重准晶排列树枝状负磁 导率材料, 测量了微波以 $0^{\circ}, 30^{\circ}$ 和 $45^{\circ}$ 平行入射时, 各组负磁导率材料及其与金属丝阵列复合的左手材 料微波透射及反射行为. 结果表明，树枝单元以准晶 分布排列能增强或减小材料整体的电磁耦合模式; 随着准晶分布旋转对称重数的增加, 材料的谐振峰 随入射角度的变化影响减小. 利用准晶树枝状负磁 导率材料的高度对称性, 将对减小左手材料的损耗 以及各向同性左手材料的实现提供新的途径.

\section{参考文献}

1 Veselago V G. The electrodynamics of substances with simultaneously negative values of permittivity and permeability. Sov Phys Usp, 1968, 10(4): 509-514

2 Pendry J B, Holden A J, Stewart W J, et al. Extremely low frequency plasmons in metallic meso structures. Phys Rev Lett, 1996, 76(25): 4773-4776[DOI]

3 Pendry J B, Holden A J, Robbins D J, et al. Magnetism from conductors and enhanced nonlinear phenomena. IEEE Trans Microwave Theor Techniq, 1999, 47(11): 2075-2084[DOI]

4 Shelby R A, Smith D R, Schultz S. Experimental verification of a negative index of refraction. Science, 2001, 292: 77-79[DOI]
5 Pendry J B. Negative refraction makes a perfect lens. Phys Rev Lett, 2000, 85: 3966-3969[DOI]

6 Fang N, Lee H, Sun C, et al. Sub-diffraction-limited optical imaging with a silver superlens. Science, 2005, 308: 534-537[DOI]

7 Pendry J B, Schurig D, Smith D R. Controlling electromagnetic fields. Science, 2006, 312: 1780-1782[DOI]

8 赵乾, 赵晓鹏, 康雷, 等. 一维负磁导率材料中的缺陷效应. 物 理学报, 2004, 53(7): 2206-2210

9 Zhang F L, Zhao Q, Liu Y H, et al. Behavior of hexagon split ring resonators and left-handed metamaterials. Chin Phys Lett, 2004, 21(7): 1330-1332[DOI]

10 Zhao X P, Kang L, Song J, et al. Defect effect of split ring resonators in left-handed metamaterials. Phys Lett A, 2005, 346: 87-91[DOI]

11 Zhu W R, Zhao X P, Ji N. Double bands of negative refractive index in the left-handed metamaterials with asymmetric defects. Appl Phys Lett, 2007, 90: 011911—011913 [DOI]

12 Song J, Zhao W, Fu Q H, et al. Two-peak property in asymmetric left-handed metamaterials. J Appl Phys, 2007, 101: $23702-$ 023706[DOI]

13 Zhang S, Fan W J, Panoiu N C, et al. Experimental demonstration of near-infrared negative-index metamaterials. Phys Rev Lett, 2005, 95: $1374041-1374044$

14 Zhou J F, Zhang L, Tuttle G, et al. Negative index materials using simple short wire pairs. Phys Rev B, 2006, 73: 0411011—0411014

15 姚远, 赵晓鹏, 赵晶, 等. 非对称开口六边形谐振单环的微波透 射特性. 物理学报, 2006, 55: 6435-6440

16 Zhou X, Fu Q H, Zhao J, et al. Negative permeability and subwavelength focusing of quasi-periodic dendritic cell metamaterials. Opt Express, 2006, 14: 7188 - 7197[DOI]

17 Liu H, Zhao X P. Magnetic response of dendritic structures at infrared frequencies. Solid State Commun, 2006, 140: 9-13[DOI]

18 Smith D R, Pendry J B, Wiltshire M C K. Metamaterials and negative refractive index. Science, 2004, 305: 788 - 792[DOI]

19 Veselago V G, Narimanov E E. The left hand of brightness: past, present and future of negative index materials. Nature Materials, 2006, 5: 759-762

20 Philippe G B, Olivier J F. Electromagnetic resonances in individual and coupled split-ring resonators. J Appl Phys, 2002 92: 2929-2932[DOI] 